\title{
Electrically small multiband antenna based on spoof localized surface plasmons
}

\author{
Rong Lin Shao ${ }^{1}$, Bo $\mathrm{Li}^{2}$, Liu Yang ${ }^{1}$, and Yong Jin Zhou ${ }^{1,3, *}$ \\ ${ }^{1}$ Key Laboratory of Specialty Fiber Optics and Optical Access Networks, Joint International Research Laboratory of Specialty \\ Fiber Optics and Advanced Communication, Shanghai Institute for Advanced Communication and Data Science, Shanghai \\ University, Shanghai 200444, PR China \\ ${ }^{2}$ Kuang-Chi Institute of Advanced Technology, State Key Laboratory of Metamaterial Electromagnetic Modulation Technology, \\ Shenzhen 518057, Guangdong, PR China \\ ${ }^{3}$ State Key Laboratory of Transducer Technology, Chinese Academy of Sciences, Shanghai 200050, PR China
}

Received: 15 September 2018 / Accepted: 29 January 2019

\begin{abstract}
Here an electrically small multiband antenna based on spoof localized surface plasmons (LSPs) has been proposed using corrugated ring resonator printed on a thin dielectric substrate with complementary metallic spiral structure (MSS) on the ground plane. It has been found that the resonant frequencies of spoof LSPs redshift by tuning the arm length of the complementary MSS, which leads to the miniaturization of the antenna. The fabricated multiband antenna has a small size of only $0.11 \lambda \times 0.1 \lambda$, covering GSM900, GSM1800, and WiFi bands. Such electrically small multiband antenna with high gain is necessary for efficient wireless energy harvesting (WEH), which can find more applications in various areas including Internet of Things (IoT), wireless sensor network (WSN), etc.
\end{abstract}

Keywords: Localized surface plasmons / metamaterials / surface plasmons

Self-sustainable low-power electronic devices have attracted significant attention in the past few years, due to their wide applications in the Internet of Things (IoT), green cellular networks [1], and wireless sensor networks (WSNs) [2]. Nevertheless, it requires smaller and smaller nodes for comfortable user experience $[2,3]$. Smaller nodes imply smaller batteries, creating strict trade-offs between the consumed energy and the performance. The energy harvesting $(\mathrm{EH})$ technologies provide a solution to replacing or recharging the battery [4]. Wireless energy harvesting (WEH) is one of the most favorable $\mathrm{EH}$ technologies for supplying continuous power to the self-sustainable stand-alone devices [2], which harvests the electromagnetic (EM) waves in the air. WEH has been applied to health monitoring sensors [5], structural health monitoring sensors [6], satellite health monitoring [7], RFID tags [8], biotelemetry communication [9], etc. Compared with the WEH systems with dedicated radio frequency $(\mathrm{RF})$ sources in references [10-14], ambient EM signals in the air are normally available at low levels ( -25 to approximately $-7 \mathrm{dBm} / \mathrm{m}^{2}$ ) [15], distributed in multiple frequency

\footnotetext{
* Corresponding author: yjzhou@shu.edu.cn
}

bands and random incident angles. To maximize the overall microwave-to-DC conversion efficiency, the designed rectenna should harvest ambient EM energy from different frequency bands simultaneously and the beam width should be wider. Besides, compared with the rectifier circuit, power management system and sensor modules, the size of antenna is too large [16], since the dimensions of conventional antennas are generally half wavelength.

Metamaterials are composed of subwavelength particles, which have been used to harvest EM energies in the microwaves regime, including a flower-like structure composed of four electrically small split-ring resonators (SRRs) [17], a circular slotted truncated corner square patch radiator placed on reactive impedance surface (RIS) [18], a parallel connection of five SRRs loaded with embedded devices [19], etc. However, previous designs focused on a single narrow frequency band and required a relatively high input power level. Loop antennas over artificial magnetic conductor surface for dual-band energy harvesting have been proposed [20]. But the antenna is not compact enough. Surface plasmons (SPs) are collective electron-photon oscillations tightly attached to the surface of a metal, which are characterized with subwavelength confinement and field enhancement [21]. SPs could be 


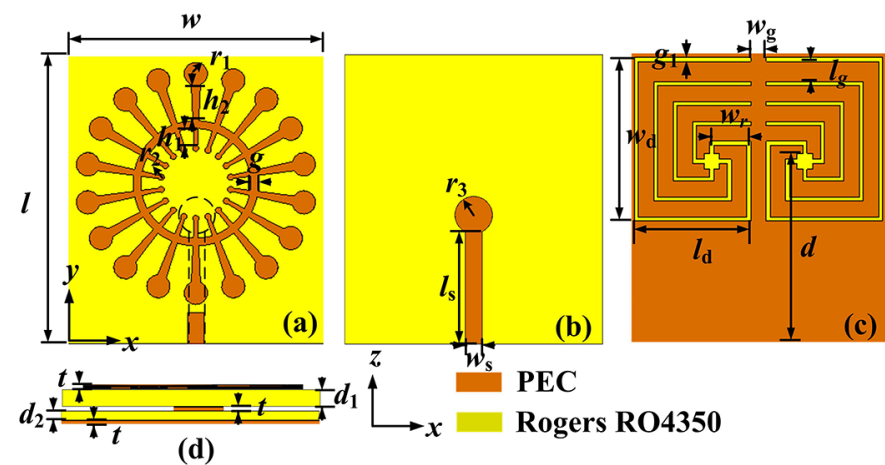

Fig. 1. (a) Top metallic layer, (b) middle metallic layer, (c) bottom metallic layer, and (d) side view of the antenna. Dimensions: $l=37 \mathrm{~mm}, w=33 \mathrm{~mm}, h_{1}=2.5, h_{2}=6 \mathrm{~mm}, g=1 \mathrm{~mm}$, $l_{\mathrm{s}}=14.34 \mathrm{~mm}, w_{\mathrm{s}}=2.1 \mathrm{~mm}, r_{1}=1.5 \mathrm{~mm}, r_{2}=0.4 \mathrm{~mm}, l_{\mathrm{d}}=15 \mathrm{~mm}$, $w_{\mathrm{d}}=20.5 \mathrm{~mm}, g_{1}=0.5 \mathrm{~mm}, w_{\mathrm{g}}=2 \mathrm{~mm}, l_{\mathrm{g}}=2.5 \mathrm{~mm}, w_{\mathrm{r}}=5.25 \mathrm{~mm}$, $d=24 \mathrm{~mm}, d_{1}=2.03 \mathrm{~mm}, d_{2}=1.01 \mathrm{~mm}$, and $t=0.018 \mathrm{~mm}$.

either propagating (surface plasmon polaritons (SPPs)) in extended interfaces [22] or localized (localized surface plasmons (LSPs)) in finite metal particles [23]. However, the electric field slightly penetrates the metal at microwave and terahertz $(\mathrm{THz})$ frequencies, which are far below the metal's plasma frequency. It has been shown that structured metal surfaces (plasmonic metamaterials) can support spoof surface plasmon (SP) modes, since the electric fields can effectively go further into the metal side [24]. Since spoof SPs can achieve subwavelength confinements to the EM waves, they have important potential applications in the miniaturization of spoof plasmonic circuits. Many antennas based on spoof SPs have been demonstrated $[25,26]$. Although the near-field characteristics of spoof LSPs have been fully investigated [27,28], the far-field characteristics are still unknown. Recently, a subwavelength unidirectional antenna was designed by combining two spoof LSPs resonators [29]. We have proposed a spoof plasmonic antenna for mobile healthcare that is composed of an annular ring slot and periodic array of T-shaped grooves [30].

In this article, an electrically small multiband antenna based on spoof LSPs is presented, which works at 0.87 , $1.34,1.75,2.45$, and $2.68 \mathrm{GHz}$, covering GSM900, GSM1800, and WiFi band. The antenna is composed of a corrugated ring resonator printed on a thin dielectric substrate and a complementary metallic spiral structure (MSS) on the ground plane. It has been found that the resonant frequencies of spoof LSPs redshift by tuning the arm length of the complementary MSS, which enables the further miniaturization of the antenna. The measurements show that the gain of the miniaturized antenna is still relatively high.

The structure of the proposed electrically small multiband antenna is shown in Figure 1. The corrugated ring resonator (the top metallic layer in Fig. 1a) is printed on a thin dielectric substrate (Rogers RO4350) with dielectric constant of 4.4 and loss tangent of 0.004 . The substrate thickness $d_{1}$ is $2.032 \mathrm{~mm}$ and $d_{2}$ is $1.016 \mathrm{~mm}$. The dimensions of the antenna are $w=33 \mathrm{~mm}$ and $l=37 \mathrm{~mm}$. It is fed through a $50-\Omega$ microstrip line (the middle metallic layer in Fig. 1b) with line length of $l_{\mathrm{s}}=16.5 \mathrm{~mm}$ and line width of $w_{\mathrm{s}}=2.1 \mathrm{~mm}$. On the bottom ground plane, a complementary MSS (the bottom metallic layer in Fig. 1c) is etched on the ground to realize the multiband and miniaturization.

Three antennas based on different structures are shown in Figure 2, where Structure 1 is the conventional ring structure and Structures 2 and 3 are the corrugated rings with periodic array of rectangle stubs and dumbbell-shaped patches, respectively. These structures are also multilayer boards, where the middle metallic layer is the $50-\Omega$ microstrip line and the bottom layer is the ground plane. To form resonances in the circular resonator antenna, the well-known requirement to be satisfied is $L \approx n \times \lambda_{\mathrm{n}}$, where $L$ is the circumference of the slot ring, $\lambda_{n}$ is the guided wavelength, and $n$ is the mode number. The standing wavelength at the resonance modes can be calculated by $\lambda_{\mathrm{n}}=2 \pi / \beta_{n}$ and $\beta_{n}$ is the plasmonic wave vector, which can be obtained from the dispersion curves.

The dispersion curves for these three kinds of structures are calculated by using the commercial software CST Microwave Studio, which are plotted in Figure 3a. It can be seen that the dispersion curve is lower when the dumbbellshaped patches are added to the ring. That is, the operation frequency of Structure 3 is the lowest when the wave vector is fixed, since its dispersion curve is the lowest. The corresponding simulated reflection coefficients of these three structures are plotted in Figure 3b. It shows that all resonant frequencies of Structure 3 redshift, which indicates that the spoof LSPs can work at lower frequency. Hence, its electrical size is smaller.

In order to make the antenna electrically smaller, complementary MSSs are etched on the ground plane. The normal complementary metallic spiral is shown in Figure $4 \mathrm{a}$, where the length of the spiral arms is the same. The proposed complementary metallic spiral composed of four spiral arms with different lengths is shown in Figure $4 \mathrm{~b}$, where the $50-\Omega$ microstrip line on the bottom layer is used to feed the structures. The dimension of the largest arm length $w_{\mathrm{d}}=13.25 \mathrm{~mm}$ and largest width $l_{\mathrm{d}}=11.25 \mathrm{~mm}$, air gap $l_{\mathrm{g}}=0.5 \mathrm{~mm}$ with inner square patch $L_{\mathrm{s}}=W_{\mathrm{s}}=1 \mathrm{~mm}$ is used in this design. The simulated reflection coefficients of the normal complementary metallic spiral and the proposed complementary metallic spiral are plotted in Figure 4c. It can be seen that more resonant modes on the proposed complementary MSS can be excited. The lowest resonant frequency of $0.98 \mathrm{GHz}$ at $\mathrm{m}_{1}$ mode is obviously lower than that of $1.78 \mathrm{GHz}$ at $\mathrm{M}_{1}$ mode for the normal complementary metallic spiral, which indicates that it can be used to make the antenna miniaturized. In order to investigate the physical mechanisms of the proposed complementary metallic spiral, Figures $4 \mathrm{~d}$ and $4 \mathrm{e}$ demonstrate the electric field distributions on the plane $1 \mathrm{~mm}$ above the structure. From Figure $4 \mathrm{~d}$, the electric field distribution of the modes $\mathrm{M}_{1}$ and $\mathrm{M}_{2}$ at 1.78 and $2.03 \mathrm{GHz}$ are similar to that of twisted electric dipole (termed as electric LSPs) and the electric field distribution of the mode $\mathrm{M}_{3}$ at $3.71 \mathrm{GHz}$ is akin to that of a magnetic dipole (termed as magnetic LSPs). From Figure $4 \mathrm{e}$, we can see that all of these modes are electric 


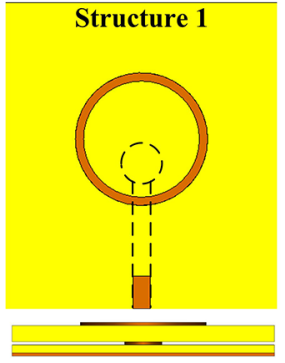

(a)

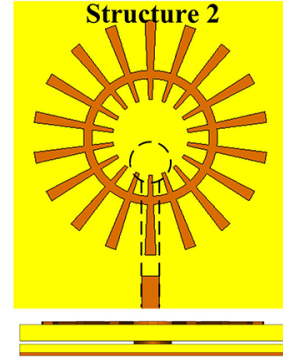

(b)

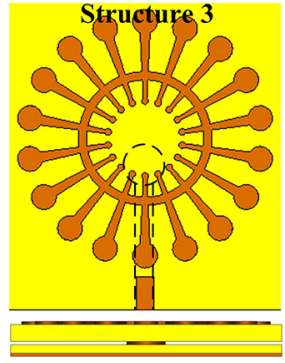

(c)

Fig. 2. The top view and side view of antennas based on (a) Structure 1, (b) Structure 2, and (c) Structure 3.
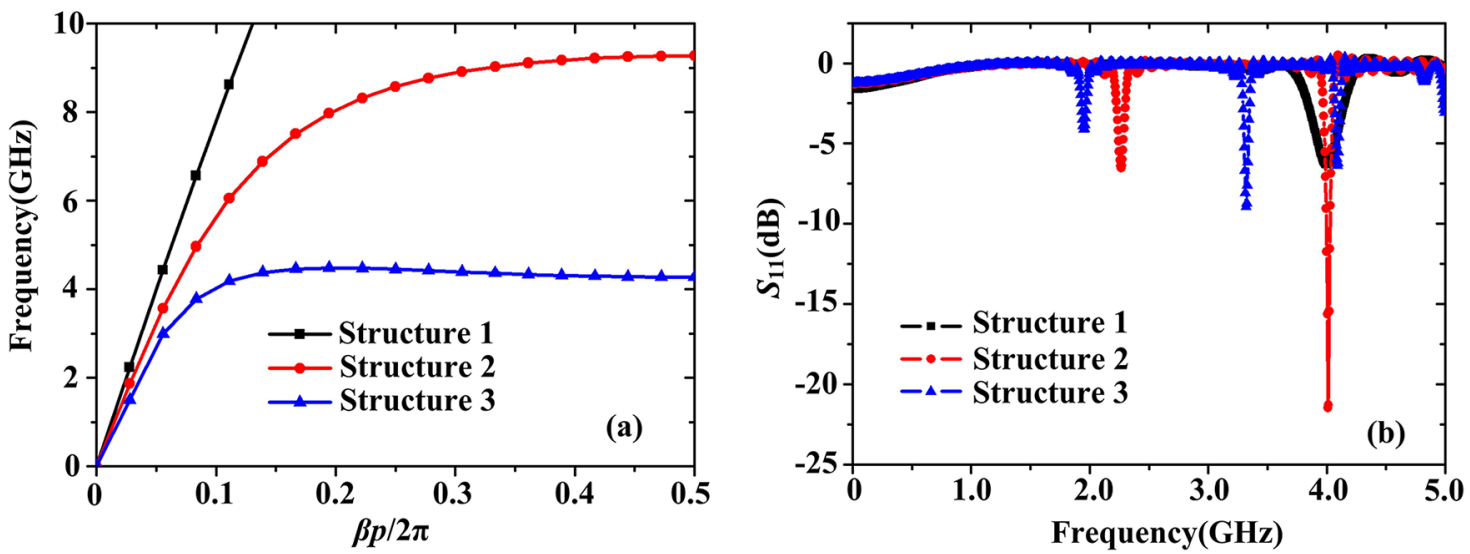

Fig. 3. (a) Dispersion curves and (b) simulated reflection coefficients of Structures 1-3.

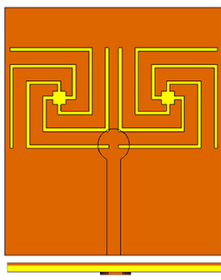

$\overline{(a)}$

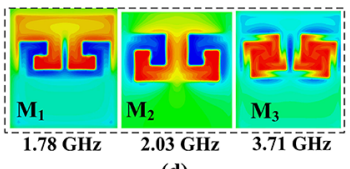

(d)

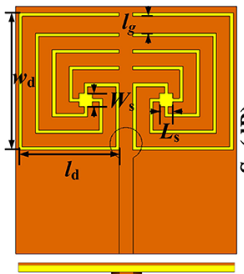

$\overline{\text { (b) }}$

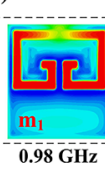

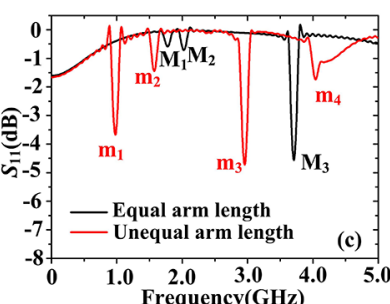

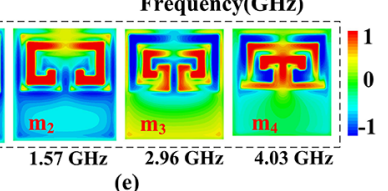

Fig. 4. Top view and side view of the complementary MSS with (a) equal arm length and (b) unequal arm length. (c) Simulated reflection coefficients of the complementary MSSs. (d) The simulated 2D $E_{z}$-field distributions on the plane located $1 \mathrm{~mm}$ above the complementary MSS with equal arm length. (e) The simulated 2D $E_{z}$-field distributions on the plane located $1 \mathrm{~mm}$ above the complementary MSS with unequal arm length.

LSPs modes and the magnetic LSPs modes are suppressed. Next, we will investigate the effects of the complementary MSS by tuning the arm length. The electric field distributions are shown in Figure 4e, from which we can see that all of these modes are electric LSPs modes and the magnetic LSPs modes are suppressed. For the lowest resonant mode $\left(\mathrm{m}_{1}\right)$, the electric fields concentrate on the longest arm. For $\mathrm{m}_{2}$ mode, they concentrate on the second longest arms. For $\mathrm{m}_{3}$ mode, they concentrate on the second shortest arms. That is, by tuning the arm length, there would be more electric LSPs modes, which is beneficial to realize the multiband and miniaturization of the antenna.

Based on the above analysis, the complementary MSS with unequal arm length has been integrated with the corrugated ring to realize the antenna miniaturization. The proposed antenna radiates through the interaction of spoof LSPs and complementary MSSs, which can work at the lower frequencies, especially due to the introduction of the complementary MSSs. Figure 5a shows the simulated reflection coefficients of the antenna with and without the complementary MSS with unequal arm length. It shows that the antenna with the complementary MSS with unequal arm length exhibits resonances at $0.88,1.32,1.74$, and $2.44 \mathrm{GHz}$. Comparing to the antenna without the complementary MSS with unequal arm length, the antenna can excite more resonant modes at lower frequencies, since the complementary MSS with unequal arm length etched on the ground plane can excite more spoof electric LSPs modes. The fabricated antenna is shown in the inset of Figure 5b and the simulated and measured $\left|S_{11}\right|$ (reflection coefficients) is represented in Figure 5b. It shows that the measured and simulated reflection coefficients are in good agreements. 

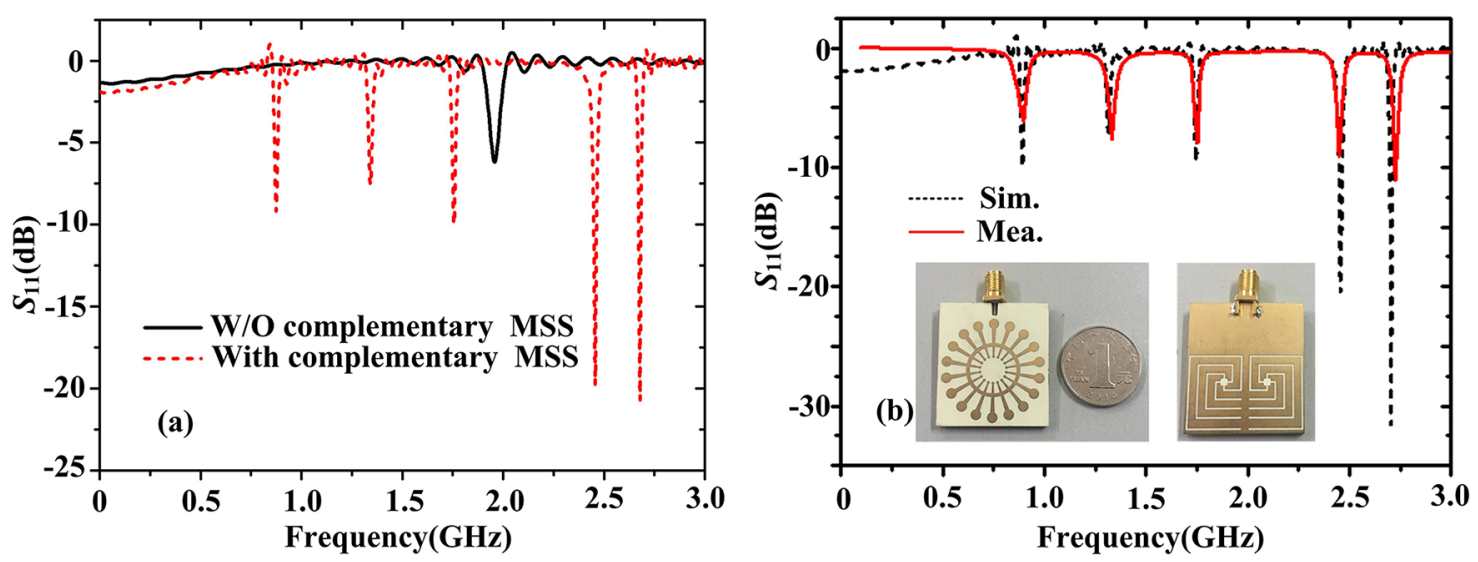

Fig. 5. (a) Simulated reflection coefficients of the antenna with and without the complementary MSS with unequal arm length. (b) Measured and simulated reflection coefficients of the fabricated antenna.
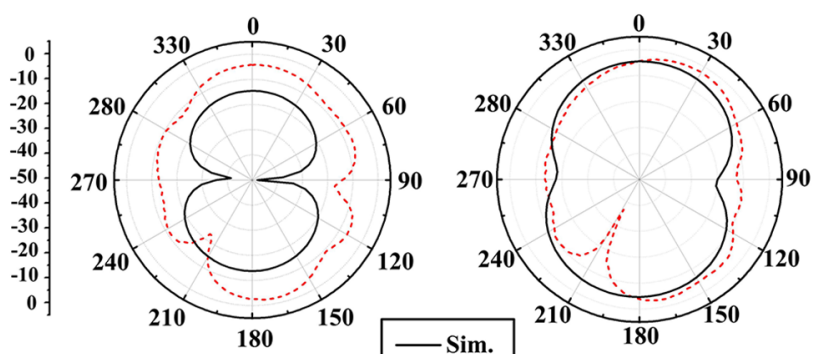

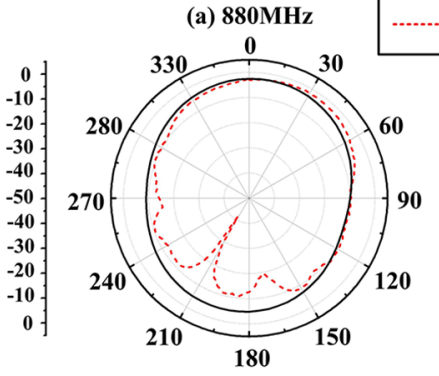

(c) $1.74 \mathrm{GHz}$
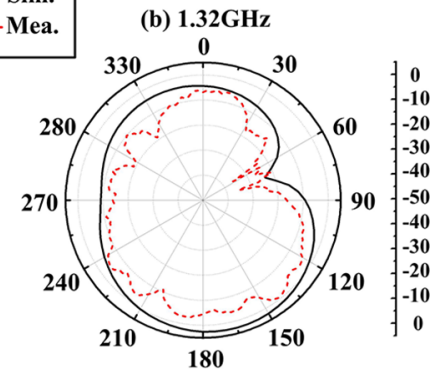

(d) $2.44 \mathrm{GHz}$
Fig. 6. Measured and simulated radiation patterns of proposed antenna.

Figure 6 gives the simulated and measured radiation patterns of the antenna at four different frequencies: 0.88 , $1.32,1.74$, and $2.44 \mathrm{GHz}$, respectively. The measured gain are $-1.53 \mathrm{dBi}$ at $0.88 \mathrm{GHz},-2.15 \mathrm{dBi}$ at $1.32 \mathrm{GHz}$, $-2.00 \mathrm{dBi}$ at $1.74 \mathrm{GHz}$, and $-2.58 \mathrm{dBi}$ at $2.44 \mathrm{GHz}$, respectively. Furthermore, it can be seen that the beamwidth of the proposed antenna is wide. There are some discrepancies between the measured and simulated results at the lowest frequency $(880 \mathrm{MHz})$, which may come from the limitations of our microwave anechoic chamber that works above $900 \mathrm{MHz}$. The performances of the proposed antenna have been compared with other electrically small multiband antennas in Table 1 . It can be observed that the proposed antenna shows smaller electrical size compared with other works in references $[15,20]$.

In this paper, an electrically small multiband antenna based on spoof LSPs has been proposed, fabricated, and
Table 1. Comparison between ours and others' design.

\begin{tabular}{llll}
\hline Reference & $\begin{array}{l}\text { Frequency } \\
(\mathrm{GHz})\end{array}$ & Size $\left(\mathrm{mm}^{2}\right)$ & Gain $(\mathrm{dBi})$ \\
\hline$[15]$ & $0.55-2.5$ & $160 \times 160$ & $2.5-5$ \\
{$[20]$} & $0.5,0.875$ & $500 \times 500$ & $8.2,8.5$ \\
This work & $0.88,1.32$, & $33 \times 37$ & $-1.53,-2.15$, \\
& $1.74,2.44$ & & $-2.00,-2.58$ \\
\hline
\end{tabular}

analyzed, and the reduction of resonant frequencies has been explained by dispersion curves. Furthermore, the complementary MSS has been etched on the ground plane, which results in further reduction of the resonant frequencies and exciting more electrical spoof LSP modes at lower frequencies. The antenna also exhibits relatively high gain with a compact size $(0.11 \lambda \times 0.1 \lambda)$. These properties make the antenna suitable for $\mathrm{WEH}$, IoT, and WSN applications.

This work was supported in part by Science and Technology Commission Shanghai Municipality (STCSM) under Grants No. 18ZR1413500 and SKLSFO2017-05, and in part by Shenzhen Science and Technology Plan under Grant No. JCYJ20151015165114938. Rong Lin Shao and Bo Li contribute equally to the work.

\section{References}

1. Z. Hasan, H. Boostanimehr, V.K. Bhargava, IEEE Commun. Surv. Tutor. 13, 524 (2011)

2. S. Kim, R. Vyas, J. Bito, K. Niotaki, A. Collado, A. Georgiadis, M.M. Tentzeris, Proc. IEEE 102, 1649 (2014)

3. S. Hemour, K. Wu, Proc. IEEE 102, 1667 (2014)

4. A. Harb, Renew. Energy 36, 2641 (2011)

5. J. Yoo, L. Yan, S. Lee, Y. Kim, H.J. Yoo, IEEE J. Solid-State Circuits 45, 178 (2010)

6. X.Y. Wang, A. Mortazawi, IEEE Trans. Microw. Theory Tech. 62, 1067 (2014) 
7. A. Takacs, H. Aubert, S. Fredon, L. Despoisse, H. Blondeaux, IEEE Trans. Microw. Theory Tech. 62, 1090 (2014)

8. A. Shameli, A. Safarian, A. Rofougaran, M. Rofougaran, F.D. Flaviis, IEEE Trans. Microw. Theory Tech. 55, 1089 (2014)

9. F. Huang, C. Lee, C. Chang, L. Chen, T. Yo, C. Luo, IEEE Trans. Antennas Propag. 59, 2646 (2011)

10. J. Yoo, L. Yan, S. Lee, Y. Kim, H.J. Yoo, IEEE J. Solid-State Circuits 45, 178 (2010)

11. F. Huang, C. Lee, C. Chang, L. Chen, T. Yo, C. Luo, IEEE Trans. Antennas Propag. 59, 2646 (2011)

12. S. Mandal, L. Turicchia, R. Sarpeshkar, IEEE Pervasive Comput. 9, 1536 (2009)

13. X. Zhang, H. Jiang, L. Zhang, C. Zhang, Z. Wang, X. Chen, IEEE Trans. Biomed. Circuits Syst. 4, 11 (2010)

14. L. Xia, J. Cheng, N.E. Glover, P. Chiang, IEEE J. Solid-State Circuits 49, 1345 (2014)

15. C. Song, Y. Huang, P. Carter, J. Zhou, S. Yuan, Q. Xu, IEEE Trans. Antennas Propag. 64, 3160 (2016)

16. K. Agarwal, T. Mishra, M.F. Karim, Nasimuddin, M.O.L. Chuen, Y.X. Guo, S.K. Panda, IEEE MTTS Int. Microw. Symp. Digest 1-4 (2013)

17. M.R. AlShareef, O.M. Ramahi, Appl. Phys. Lett. 104, 2359 (2014)
18. K. Agarwal, T. Mishra, M.F. Karim, Nasimuddin, M.O.L. Chuen, Y.X. Guo, S.K. Panda, IEEE Microw. Symp. Digest 1-4 (2014)

19. A.M. Hawkes, A.R. Katko, S.A. Cummer, Appl. Phys. Lett. 103, 163901 (2013)

20. H. Kamoda, S. Kitazawa, N. Kukutsu, K. Kabayashi, IEEE Trans. Antennas Propag. 63, 4408 (2015)

21. E. Ozbay, Science 311, 189 (2006)

22. W.L. Barnes, A. Dereux, T.W. Ebbesen, 424, 824 (2010)

23. Y. Sonnefraud, A.L. Koh, D.W. Mccomb, S.A. Maier, Laser Photonics Rev. 6, 277 (2012)

24. J.B. Pendry, L. Martín-Moreno, F.J. Garcia-Vidal, Science 305, 847 (2004)

25. J.J. Wu, D.J. Hou, H.L. Chiueh, J.Q. Shen, C.J. Wu, Y.H. Kao, W.C. Lo, T.J. Yang, C.J. Wu, Electron. Lett. 50, 1611 (2014)

26. A. Kianinejad, Z.N. Chen, C.W. Qiu, IEEE Trans. Antennas Propag. 65, 681 (2017)

27. A. Pors, E. Moreno, L. Martin-Moreno, J.B. Pendry, F.J. Garcia-Vidal, Phys. Rev. Lett. 108, 223905 (2012)

28. X. Shen, T.J. Cui, Laser Photonics Rev. 8, 137 (2014)

29. F. Qin, Q. Zhang, J.J. Xiao, Sci. Rep. 6, 29773 (2016)

30. L. Yang, Y.J. Zhou, C. Zhang, X.M. Yang, X.-X. Yang, C. Tan, IEEE J. Electromagn. RF Microw. Med. Biol. 2, 1 (2018)

Cite this article as: Rong Lin Shao, Bo Li, Liu Yang, Yong Jin Zhou, Electrically small multiband antenna based on spoof localized surface plasmons, EPJ Appl. Metamat. 6, 11 (2019) 